$\mathrm{XX}$ Міжнародний симпозіум «Методи дискретних особливостей в задачах математичної фізики/Discrete Singularities Methods in Mathematical Physics»,

УДК 533.6.013.16:533.6.013.02 МДОЗМФ/DSMМРh-2021

MSC 76G25

\title{
Inertial-circulating principle of swimming and flight of hydro- and aerobionts. Part 1
}

\author{
A.V. Shekhovtsov \\ Institute of Hydromechanics NASU, Kyiv, Ukraine \\ E-mail: avshekhovtsov@gmail.com
}

\begin{abstract}
For the case of modeling in the nonlinear ideal formulation of oscillations of an infinitely thin profile of the wing-propulsion, three components of the thrust force coefficient were distinguished - inertial, circulating, and vortex. The contribution to the traction force of each of the obtained components is investigated and the mechanisms of wing traction force formation at different types of oscillations are explained. It is revealed that the inertial-circulating principle underlies the work of the wing-propulsion. The inductive effect of the vortex trail on the traction force is small and negative.
\end{abstract}

Key words: Inertia-circulation principle, swimming, flight, thrust, oscillation of the wing-propulsion.

\section{Инерционно-циркуляционный принцип плавания и полёта гидро- и аэробионтов. Часть 1}

\author{
А.В. Шеховцов \\ Институт гидромеханики НАНУ, Киев, Украина \\ E-mail:avshekhovtsov@gmail.com
}

\begin{abstract}
Для случая моделирования в нелинейной идеальной постановке колебаний бесконечно тонкого профиля крыла-движителя выделено три компоненты коэффициента силы тяги - инерционная, циркуляционная и вихревая. Исследован вклад в силу тяги каждой из полученных компонент и объяснены механизмы образования силы тяги крыла при различных видах колебаний. Обнаружено, что в основе работы крыладвижителя лежит инерционно-циркуляционный принцип. При этом индуктивное влияние вихревого следа на силу тяги мало и негативно.
\end{abstract}

Ключевые слова: Инерционно-циркуляционный принцип, плавание, полёт, коэффициент силы тяги, колебания крыла-движителя.

\section{1. Введение}

Со времен первых исследований по колеблющемуся крылу-движителю и до настоящего момента вопрос о природе сил, возникающих на крыле, оставался открытым, несмотря на довольно развитые аналитические и численные методы их определения. Так, например, в классической работе Голубева [1] утверждается: «...взмахами машущего крыла мы вызываем образование за ним обращенной дорожки Бенара-Кармана, которая в свою очередь создает силу, направленную в сторону, противоположную той, которая возникает от обыкновенной дорожки, т.е., силу тяги.» Подобное толкование природы силы тяги крыла-движителя можно встретить также у Ньюмана, Ву [2], Рейнера [3] и в некоторых других работах современных исследователей.

Представляется, что при этом происходит перемена причины и следствия местами, то есть, вихревой след не является причиной возникновения сил на машу- 
щем крыле. На формирование ложной точки зрения могли повлиять следующие обстоятельства. Во-первых, импульс, уносимый каждой новой парой вихрей по истечении очередного периода колебаний, численно равен (для идеальной среды) импульсу, приобретаемому крылом за этот период. Во-вторых, скорости от двойного ряда вихрей с противоположными направлениями вращения складываются вдоль направления поступательного перемещения крыла, создавая течение, напоминающее реактивную струю.

Как известно, помимо наиболее распространенного метода непосредственного расчета реакции потока на крыло, существует косвенный метод, основанный на применении теоремы Эйлера количества движения жидкого объема сплошной среды. На этом методе основана теория “активного диска”, применяемая при подсчете реакции струи, создаваемой системой вращающихся лопастей. Теория “активного диска" использует то обстоятельство, что создаваемый упор имеет реактивную (инерционную) природу - лопасти отбрасывают назад струю, границу которой можно условно принять непрерывной и стационарной. Данный метод, кроме его приближенности, имеет еще один существенный недостаток: в силу своего интегрального характера он оставляет за скобками весь процесс создания нагрузок на крыле-движителе и, как следствие, процесс образования вихревого следа.

Поэтому для выяснения природы сил, возникающих на крыле-движителе, необходимо применять именно методы, позволяющие непосредственно рассчитывать нагрузки на крыле. Для таких методов в предельном случае бесконечно тонкого крыла, заменяющего реальное крыло конечной толщины, теоретически возможно представление главного вектора внешних сил крыла в виде суммы нормальной и подсасывающей сил. При этом условия обтекания кромок будут различными: переднюю кромку среда должна беспрепятственно обтекать, что в случае ее бесконечно большой кривизны приводит к возникновению кинематической особенности вида $1 / \sqrt{x}$ (бесконечной скорости перетекания потока через переднюю кромку крыла) и, соответственно, к возникновению области разрежения. С задней же кромки крыла должен реализовываться сход вихревой пелены, при котором перетекание потока, а, следовательно, и бесконечные скорости, не реализуются (условие Кутта-Жуковского-Чаплыгина). В случае реального достаточно тонкого крыла величины разрежений и скоростей вблизи передней кромки, хотя и велики, но все же конечны, а подсасывание, как и нормальная сила, создается за счет перепада давления на противоположных кромках с весьма малыми, но конечными площадями. Причины возникновения перепада давления могут быть следующие: за счет мгновенных присоединенных масс (инерционная компонента), за счет индуктивных скоростей от вихревой системы, порожденной крылом (вихревая компонента) и за счет относительной невозмущенной скорости потока (циркуляционная компонента). Подобное разделение сил для случая бесконечно малой амплитуды колебаний бесконечно тонкого крыла было осуществлено еще Карманом и Сирсом [4], однако линейная постановка задачи давала возможность исследовать лишь вырожденный случай, когда вихревой след находится в плоскости крыла, в то время как амплитуды колебаний крыльев гидро- и аэробионтов сопоставимы с их хордами. Попытаемся выделить эти компоненты, соответствующие силам различной природы и рассмотрим величину их вклада в силу тяги крыла-движителя при различных режимах его работы для нелинейного случая. 
XX Міжнародний симпозіум «Методи дискретних особливостей в задачах математичної фізики/Discrete Singularities Methods in Mathematical Physics», МДОЗМФ/DSMМPh-2021

\section{2. Постановка задачи о нелинейных колебаниях крыла-движителя}

Пусть среда является сплошной и обладает свойствами идеальности, несжимаемости и невесомости (отсутствуют внешние объемные силы). Во всех точках среды в начальный момент времени предполагается отсутствие вихрей, а сама среда неподвижной.

Будем предполагать, что крыло $S$ является бесконечно тонким, непроницаемым и нерастяжимым. Примем к рассмотрению модель циркуляционного обтекания крыла со сходом слоя потока с его поверхности, а также постулат КуттаЖуковского-Чаплыгина о конечности скорости потока в окрестности задней кромки крыла. В силу принятых свойств среды и крыла, с задней кромки движущегося крыла будет сходить вихревая пелена $\sigma$, точки которой будут двигаться по траекториям жидких частиц, то есть она будет вморожена в потенциальную среду. Скачок потенциала скорости $\Delta \Phi$ на свободной вихревой пелене $\sigma$ не будет меняется со временем.

Принятая модель циркуляционного обтекания, допускающая образование свободной вихревой пелены за крылом в процессе его движения, не противоречит теореме Лагранжа и динамической теореме Кельвина, так как в этих теоремах сохранение завихренности и циркуляции выполняется не для области пространства, а для жидкого объема среды. При этом границей рассматриваемой среды $\Omega$ является совокупность $S$ и $\sigma$, причем в область $\Omega$ сами $S$ и $\sigma$ не входят.

Сформулируем начально-краевую задачу [5].

Уравнение Лапласа для потенциала $\Phi$ :

$$
\operatorname{divgrad} \Phi(x, y, t)=0 \quad t>0,(x, y) \in \Omega
$$

Запишем граничные условия для уравнения Лапласа.

Условие непроницаемости крыла $S$ :

$$
\begin{array}{r}
\lim _{(x, y) \rightarrow\left(x_{0}, y_{0}\right)} \vec{n}_{S}\left(x_{0}, y_{0}, t\right) \cdot \operatorname{grad} \Phi(x, y, t)=\vec{n}_{S}\left(x_{0}, y_{0}, t\right) \cdot \vec{W}^{*}\left(x_{0}, y_{0}, t\right) \\
\forall t,\left(x_{0}, y_{0}\right) \in S \backslash\left(B_{1} \cup B_{2}\right),(x, y) \in \Omega,
\end{array}
$$

где $\vec{W}^{*}$ - вектор скорости точек крыла; $B_{1}$ - задняя кромка крыла: $B_{2}-$ передняя кромка крыла: $\vec{n}_{S}-$ вектор нормали к крылу.

Условие движения вихревой пелены $\sigma$ по траекториям жидких частиц:

$$
\lim _{(x, y) \rightarrow\left(x_{0}, y_{0}\right)} \operatorname{grad} \Phi(x, y, t)=\overrightarrow{\tilde{W}}\left(x_{0}(t, \tilde{t}), y_{0}(t, \tilde{t}), t\right) \quad t \geq \vec{t},\left(x_{0}, y_{0}\right) \in \sigma,(x, y) \in \Omega,
$$

где $\overrightarrow{\widetilde{W}}$ - вектор скорости точек вихревой пелены; $\tilde{t}$ - время, при котором $\left(x_{0}, y_{0}\right) \in S$.

Условие постоянства скачка потенциала $\Delta \Phi$ в точках $\sigma$ :

$$
\Delta \Phi\left(x_{0}(t, \tilde{t}), y_{0}(t, \tilde{t}), t\right)=\Delta \Phi\left(x_{1}, y_{1}, \tilde{t}\right) \quad t \geq \tilde{t},\left(x_{0}, y_{0}\right) \in \sigma .
$$

Условие затухания возмущений на бесконечном удалении от крыла и вихревой пелены:

$$
\begin{array}{r}
\lim _{(x, y)-\left(x_{0}, y_{0}\right) \rightarrow \infty} \operatorname{grad} \Phi(x, y, t)= \\
\quad \lim _{(x, y)-\left(x_{0}, y_{0}\right) \rightarrow \infty} \frac{\partial \Phi(x, y, t)}{\partial t}=0 \\
\forall t,(x, y) \in \Omega,\left(x_{0}, y_{0}\right) \in S \cup \sigma .
\end{array}
$$


Как известно, внешняя смешанная краевая задача для уравнения Лапласа имеет решение с точностью до константы при условии:

$$
\oint_{S} \vec{W}^{*}\left(x_{0}, y_{0}, t\right) \cdot \vec{n}_{S}\left(x_{0}, y_{0}, t\right) d s=0 \quad \forall t, \quad\left(x_{0}, y_{0}\right) \in S .
$$

Данное условие выражает закон сохранения массы внутри границы $S$ (в общем случае - произвольной замкнутой), что в плоском случае для несжимаемой среды эквивалентно сохранению площади внутри непроницаемого контура $S$, или нулевому балансу потока через его поверхность. В силу принятых ограничений, в рассматриваемом случае это условие будет выполняться для любого момента времени, то есть существование решения задачи (1)-(6) обеспечено.

В силу того, что в плоском случае область $\Omega$ является двусвязной, циркуляция скорости по замкнутому контуру $C$, охватывающему границу $S \cup \sigma$, в общем случае может быть отлична от нуля, а потенциал $\Phi$, соответственно, многозначной функцией. Без ограничения общности, в качестве контура $C$ можно взять жидкий контур. Тогда, в соответствии с теоремой Кельвина, циркуляция по нему будет постоянной. Отсюда приходим к следующему условию обеспечения единственности решения поставленной задачи:

$$
\oint_{C} \operatorname{grad} \Phi(x, y, t) \cdot \delta \vec{r}(x, y, t)=\text { const } \quad \forall t,(x, y) \in C \in \Omega .
$$

Задача Коши для нахождения формы свободной вихревой пелены $\sigma$ в произвольный момент времени $t$ :

$$
\left\{\begin{array}{l}
\vec{r}\left(x_{0}(t, \tilde{t}), y_{0}(t, \tilde{t}), t\right)=\vec{r}\left(x_{1}, y_{1}, \tilde{t}\right), \quad t=\tilde{t}, \\
\frac{d \vec{r}\left(x_{0}(t, \tilde{t}), y_{0}(t, \tilde{t}), t\right)}{d t}=\overrightarrow{\widetilde{W}}\left(x_{0}(t, \tilde{t}), y_{0}(t, \tilde{t}), t\right), \quad t>\tilde{t},
\end{array}\right.
$$

где $\vec{r}$ - радиус-вектор произвольной точки $\left(x_{0}, y_{0}\right) \in \sigma$.

Для начально-краевой задачи (1)-(8) начальными условиями будут известная форма границ и значение потенциала в момент $t=0$ :

$$
S \cup \sigma=S, \quad \Phi(x, y, t)=0 \quad t=0,(x, y) \in \Omega
$$

Скорости точек свободной вихревой пелены, входящие в уравнения (3) и (8), являются функционалами от всех предшествующих положений границ $S$ и $\sigma$, которые необходимо находить в процессе решения задачи. Поэтому начальнокраевая задача (1)-(9) является нелинейной.

После решения кинематической задачи (1)-(9), используя интеграл КошиЛагранжа, можно определить динамические характеристики крыла и поле давления вокруг него.

\section{3. Решение задачи усовершенствованным методом дискретных вихрей}

Решение уравнения Лапласа будем искать в виде суммы потенциалов от непрерывно распределенных по границам области гидродинамических особенностей. При выборе типа особенностей будем учитывать необходимость обеспечить тангенциальный разрыв скорости на поверхности крыла и свободной вихревой пелене, а также затухание возмущений на бесконечном удалении от границ. Такими свойствами обладают потенциалы двойного слоя и слоя вихрей. Поэтому, используя какой-либо из них, останется лишь определить его плотность так, чтобы удовлетворить условию непроницаемости крыла. Подставление выражения для по- 
XX Міжнародний симпозіум «Методи дискретних особливостей в задачах математичної фізики/Discrete Singularities Methods in Mathematical Physics»,

\section{МДОЗМФ/DSMMPh-2021}

тенциала двойного слоя в граничное условие (2), с учетом параметрического представления границ, даст сингулярное интегро-дифференциальное уравнение:

$$
\begin{gathered}
\frac{1}{2 \pi} \int_{S} \frac{y_{0 l_{S}}^{\prime}\left(y_{0}-y\right)+x_{0 l_{S}}^{\prime}\left(x_{0}-x\right)}{\left(x_{0}-x\right)^{2}+\left(y_{0}-y\right)^{2}} g_{l_{S}}^{\prime}(x, y, t) d l_{S}=\vec{W}^{*}\left(x_{0}, y_{0}, t\right) \cdot \vec{n}_{S}\left(x_{0}, y_{0}, t\right)- \\
\quad-\frac{1}{2 \pi} \int_{\sigma} \frac{y_{0 l_{S}}^{\prime}\left(y_{0}-y(t, \tilde{t})\right)+x_{0 l_{S}}^{\prime}\left(x_{0}-x(t, \tilde{t})\right)}{\left(x_{0}-x(t, \tilde{t})\right)^{2}+\left(y_{0}-y(t, \tilde{t})\right)^{2}} g_{l_{\sigma}}^{\prime}(x(t, \tilde{t}), y(t, \tilde{t})) d l_{\sigma} .
\end{gathered}
$$

Сингулярные интегралы в (10) понимаются в смысле главного значения Коши. Условием единственности решения для (10) будет:

$$
\Gamma=\int_{S \cup \sigma} g_{l_{\Sigma}}^{\prime}(x, y, t) d l_{\Sigma}=0 . \quad \forall t,(x, y) \in S \cup \sigma .
$$

Задача Коши примет вид:

$$
\left\{\begin{array}{l}
\vec{r}\left(x_{0}(t, \tilde{t}), y_{0}(t, \tilde{t}), t\right)=\vec{r}\left(x_{1}, y_{1}, \tilde{t}\right), \quad t=\tilde{t}, \quad\left(x_{0}, y_{0}\right) \in \sigma \\
\frac{d \vec{r}\left(x_{0}(t, \tilde{t}), y_{0}(t, \tilde{t}), t\right)}{d t}= \\
=\frac{1}{2 \pi} \int_{S \cup \sigma} g_{l_{\Sigma}}^{\prime}(x, y, t) \frac{\left(y_{0}(t, \tilde{t})-y\right) \vec{i}-\left(x_{0}(t, \tilde{t})-x\right) \vec{j}}{\left(x_{0}(t, \tilde{t})-x\right)^{2}+\left(y_{0}(t, \tilde{t})-y\right)^{2}} d l_{\Sigma}, \\
t>\tilde{t}, \quad\left(x_{0}, y_{0}\right) \in \sigma .
\end{array}\right.
$$

Полученная система (10)-(12) - нелинейная. Поэтому, для решения поставленной задачи необходимо привлекать численные методы.

Так как скорости, индуцируемые двойным слоем, эквивалентны скоростям от соответствующего вихревого слоя, то для создания численной модели будем использовать усовершенствованный метод дискретных вихрей (УМДВ) [6]. УМДВ позволяет представить скорость любой точки среды в виде суммы невозмущенной скорости потока, возмущенной скорости от системы суммарных дискретных вихрей, моделирующих обтекаемое тело, а также возмущенной скорости от системы свободных вихрей, моделирующих непрерывную вихревую пелену, сошедшую с тела. Кроме того, в случае, когда крыло полагается бесконечно тонким, применение интеграла Коши-Лагранжа для расчета перепада давления на крыле с учетом свойств плотности вихревого слоя $\gamma$ приводит к линеаризации нелинейных слагаемых. Указанные особенности моделирования дают возможность представить коэффициент силы тяги в виде трех компонент:

- инерционной: $C_{T_{I}}(\tau)=2 \int_{S} \frac{\partial^{\prime} \Delta \varphi(s(\tau), \tau)}{\partial \tau} \cos (n(s, \tau), x) d s$, где $\Delta \varphi$ - скачок потенциала скорости на поверхности крыла $S$; штрих при частной производной означает, что дифференцирование совершается в подвижной системе координат крыла;

- циркуляционной:

$$
C_{T_{C}}(\tau)=C_{Q}(\tau) \cos \left(n\left(s_{0}, \tau\right), y\right)-2 \int_{S} \gamma(s(\tau), \tau)\left(\hat{w}_{\mu}(s(\tau), \tau)-w_{\mu}^{*}(s(\tau), \tau)\right) \cos (n(s, \tau), x) d s,
$$


где $C_{Q}-$ коэффициент подсасывающей силы; $s_{0}-$ передняя кромка; $\hat{w}_{\mu}-$ касательная проекция скорости от системы суммарных вихрей, моделирующих вихревую пелену крыла; $w_{\mu}^{*}$ - касательная проекция переносной скорости крыла;

- вихревой: $C_{T_{V}}(\tau)=-2 \int_{S} \gamma(s(\tau), \tau) \tilde{w}_{\mu}(s(\tau), \tau) \cos (n(s, \tau), x) d s$, где $\tilde{w}_{\mu}$ - касательная проекция скорости от системы свободных вихрей.

Указанное разделение сил имеет довольно ясную физическую основу. Циркуляционная составляющая есть квазистационарный аналог силы Жуковского и определяется текущим значением циркуляции по контуру, прилегающему к крылу (без учета сошедших вихрей). Инерционная составляющая есть нестационарная компонента, зависящая от текущей присоединенной массы крыла. Вихревая (индуктивная) компонента определяется текущими величиной и распределением завихренности вокруг крыла.

\section{ЛИТЕРАТУРА}

1. Голубев В.В. Избранные труды по аэродинамике. М.-Л.: ГИТТЛ, 1957. 977 с.

2. Newman J.N., Wu T.Y. Hydromechanical aspects of fish swimming. Swimming and Flying in Nature. N.Y.-L.: Plenum Press, 1975. 1. P. 615-634.

3. Reyner J.M.V. A vortex theory of animals flight. Part 1. The vortex wake of a hovering animal. J.Fluid Mech. 1979. 91, № 4. P. 697-730.

4. Karman Th., Sears W.R. Airofoil theory for non-uniform motion. J. of Aeron. Sciences. 1938. 5, № 9-10. P. 415-462.

5. Shekhovtsov A.V. Non-Linear Mathematical Model of Dolphin Tail Fin Motion. International Journal of Fluid Mechanics Research. 2001. 28, № 1/2. P. 240-265.

6. Dovgiy S.A., Shekhovtsov A.V. An Improved Vortex Lattice Method for Nonstationary Problems. Journal of Mathematical Sciences. 2001. 104, № 6. P. 1615-1627.

Надійшла 30.05.2021.

\section{Інерційно-циркуляційний принцип плавання і польоту гідро- і аеробіонтів. Частина 1}

\section{О.В. Шеховцов}

Інститут гідромеханіки НАНУ, Київ, Україна

E-mail: avshekhovtsov@gmail.com

Для випадку моделювання в нелінійній ідеальній постановці коливань нескінченно тонкого профілю крила-рушія виділено три компоненти коефіцієнта сили тяги інерційну, циркуляційну і вихрову. Досліджено внесок в силу тяги кожної з отриманих компонент і пояснено механізми утворення сили тяги крила при різ-них видах коливань. Виявлено, що в основі роботи крила-рушія лежить інерційноциркуляційний принцип. При цьому індуктивний вплив вихрового сліду на силу тяги малий і негативний.

Ключові слова: Інерційно-циркуляційний принцип, плавання, політ, коефіцієнт сили тяги, коливання крила-рушія. 